\title{
Mobile Phone Embedded With Medical and Security Applications
}

\author{
Bhaskar Kamal Baishya \\ Department of Computer Science and Engineering, North Eastern Regional Institute of Science and Technology, \\ Nirjuli, Arunachal Pradesh, India
}

\begin{abstract}
This paper introduces MOBILE PHONE EMBEDDED WITH ANDROID BASED EMERGENCY ALERT BUTTON AND MEDICAL TOOLS - a mobile phone can serve us with various functions of security applications and medical tools. This serves us with facilities of (1)ANDROID BASED EMERGENCY ALERT BUTTON, (2)ACCU-CHEK GLUCOMETER, (3)BODY MASS INDEX CALCULATOR, (4)BASAL METABOLIC RATE CALCULATOR, (5)BODY FAT CALCULATOR, (6)ACTIVITY CALCULATOR, (7)ANDROID BASED HOME SECURITY SYSTEM. Thus this embedded system will act like a self-doctor which can help us as medical equipment. Collectively the mobile phone can be used as a modern medical toolbox having all the facilities we can inherit and also it can use as for managing or analysing the daily common diseases. The application like home security system discussed here can be incorporated with mobile phones is sure to benefit every people who always have a doubt of their belongings being secure in the house in their absence. Most of the mobile devices that are manufactured nowadays in the market are ANDROID OS based. Taking this into interest the application can be developed in ANDROID and made user friendly, thereby making the applications highly robust across different mobile devices and variety of users.
\end{abstract}

Keywords: ANDROID, Short Message Service (SMS), Global Communication for mobile system (GSM).

\section{Introduction}

Usually, a cell phone has the facilities of calling, message transmission and receiving, voice and video recording, FM and AM radio, photo capturing. It also has memory card slots along with sim card slots. It can also be used as for playing different types of games. It will also have internet connectivity. Apart from these common cell phone functions it can be used for various emergency situations like an woman can use it to call for help to save her from any danger by pressing a Save Our Souls (SOS) button without any need of talking. It can also be used as medical equipment. It can also help in controlling blood pressure. It can also be used as Body mass index (BMI) calculator, Basal metabolic rate (BMR) calculator, body fat calculator, activity calculator.

\section{Application For Emergency Situations}

The security of women at night and at times even in day when travelling alone is a concern. On 16th December, 2012 New Delhi, capital of India witnessed a heinous crime. A female physiotherapy intern was beaten and gang raped by six persons. The ambulance and other service had reached the spot late hindering emergency medical treatment. It has been observed that at times the instant communication of message of one's whereabouts precisely is a problem. This paper describes about an SOS application that can be developed in android platform. The uniqueness of this application apart from other SOS application available is that the user need not spent time navigating inside the phone menu; unlock the screen, to trigger the service. He instead, can directly press the power button and thereby, popping up a SOS screen and user can directly click the SOS button triggering the application in the background, sending the location (latitude and longitude) to all the preregistered phone numbers in the application. Many applications available in the market send a custom message to the number registered but not the location of the user. In this application the longitude, latitude information and the general idea of the place of the current position of the mobile user is appended with the custom message that had been initially set in the application and is transmitted to the phone numbers registered. This feature of the application not only helps in finding the exact location of the person in problem but also will help the police to trace the location of incident at latter time easily. 


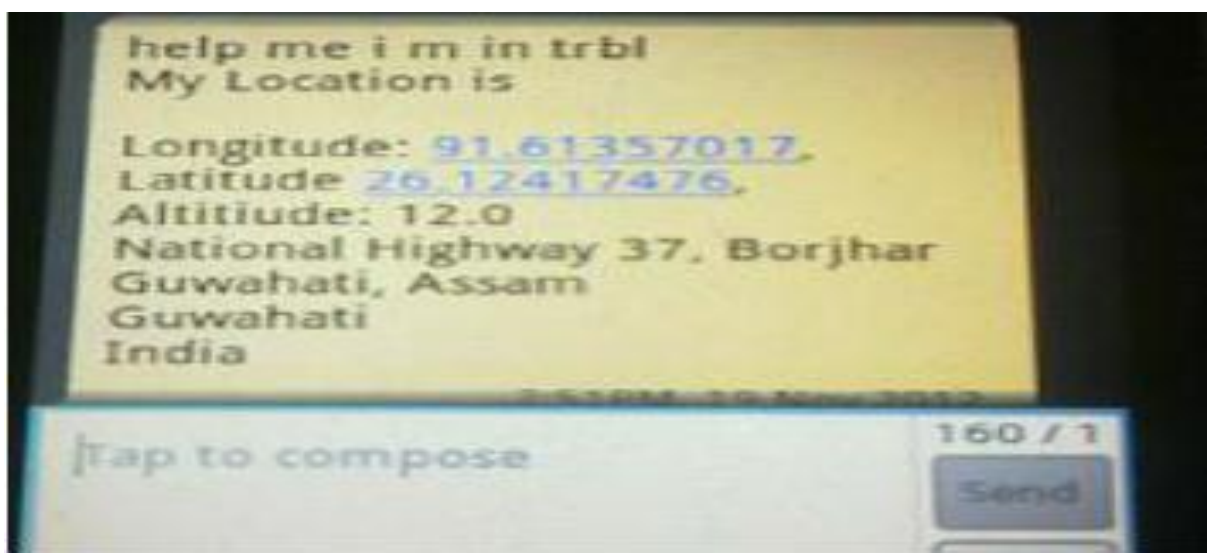

Figure 1 : Security Application screen

\section{1) Accu-Chek Glucometer}

\section{Medical Applications}

The mobile phones can also be used as for analyzing the diabetes or the sugar level. It can use the concept of "Accu-chek glucometer" for monitoring the level of sugar or the diabetes level. The original Accuchek is generally large in shape, so accu-chek cannot be directly used here since, it will not be feasible with it. So, the software used inside the device Accu-chek is used. The figure of the device accu-chek is in Figure 2.



Figure 2 : Accu-chek

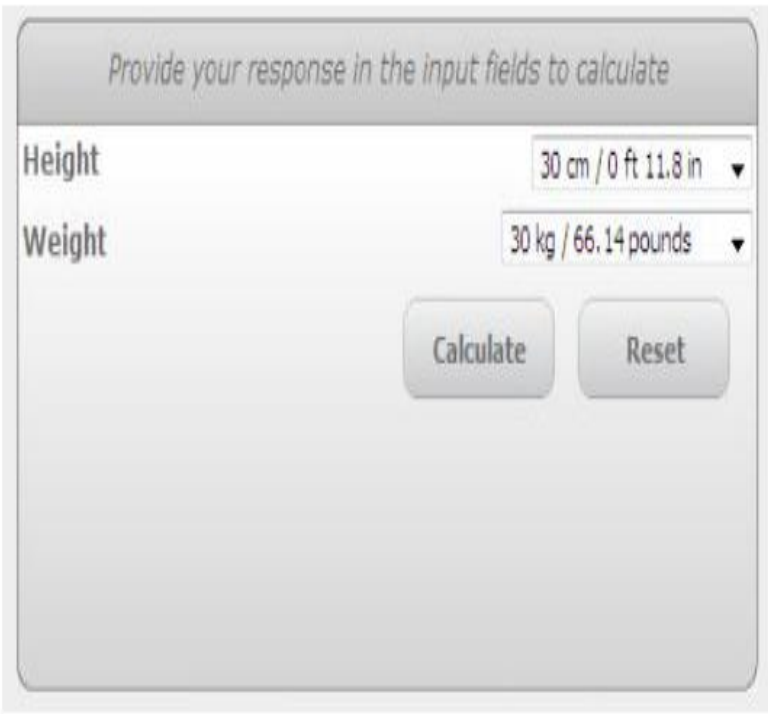

Figure 3 : BMI calculator

\section{2) Body Mass Index Calculator}

It can also be used for many health-related queries. It can be used for calculating Body Mass Index (BMI). The height and weight of the person is asked. There is fields in which a person can enter and by clicking on calculate the software will be able to calculate. A format of BMI calculator is in Figure 3. Our body mass index (BMI) is an estimate of our body fat that is based on your height and weight. Doctors use BMI, along with other health indicators, to assess an adult's current health status and potential health risks. We can determine our BMI with the calculator shown above in figure 3 .

\section{3) Basal Metabolic Rate Calculator}

It can be also used for calculating Basal Metabolic Rate (BMR). The required fields to be entered are both different for male and female since the body requirements are different for both male and female. BMR calculators for both the types are in Figure 4. 


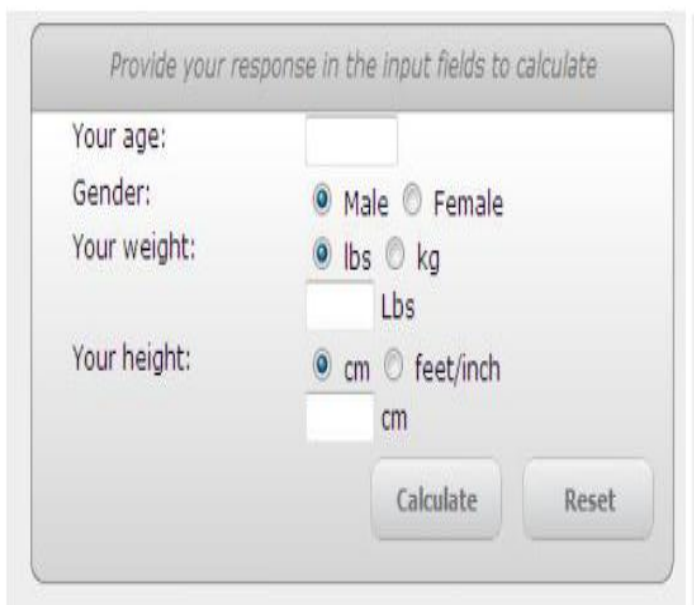

Figure 4 : BMR calculator

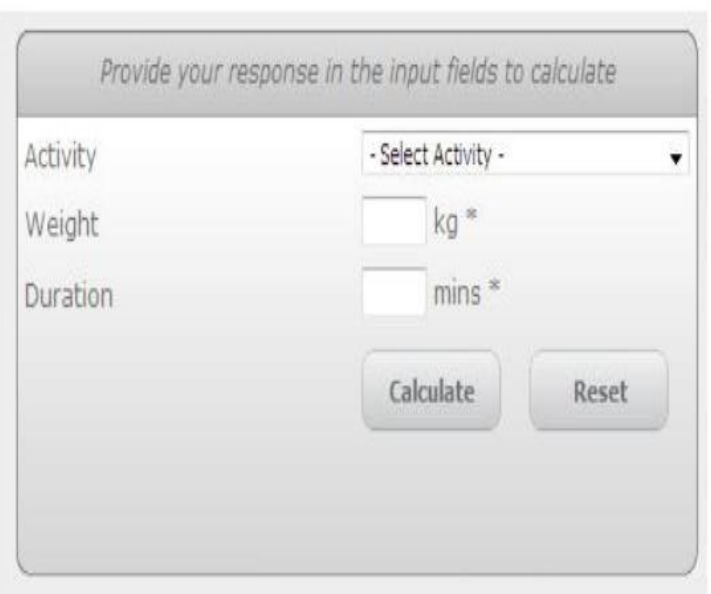

Figure 5 : Activity calculator

\section{4) Activity Calculator}

It can be used for calculating activity calculator. There is fields in which a person can enter and by clicking on calculate the software will be able to calculate. A format of activity calculator is in Figure 5.

\section{5) Body Fat Calculator}

It can be also used for calculating Body Fat Calculator. The required fields to be entered are both different for male and female since the body requirements are different for both male and female. Body fat calculator for both the types is in Figure 6 and Figure 7.

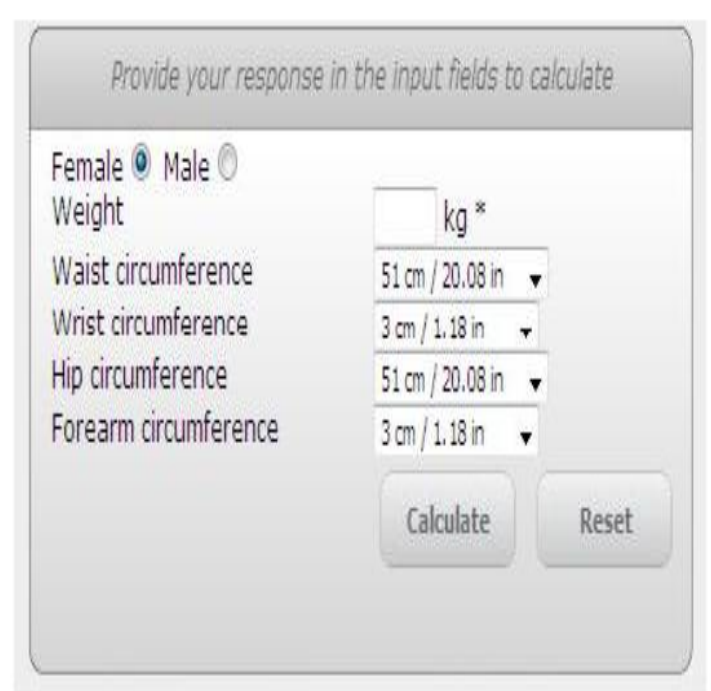

Figure 6 : Body fat calculator (for female)

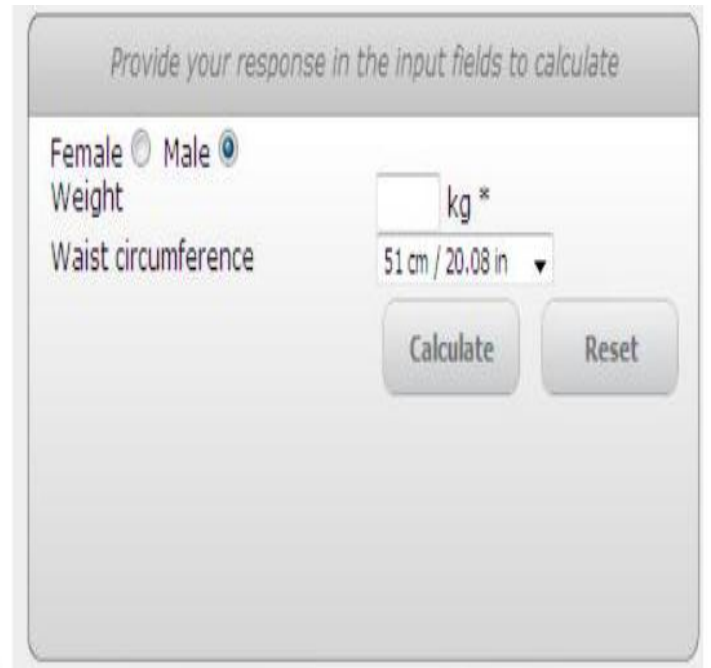

Figure 7 : Body fat calculator (for male)

\section{Home Security Application}

The security of one's belongings when a person leaves his/her house is always a concern with increasing number of incidents of theft, robbery etc. Many automated systems has been developed which informs the owner in a remote location about any intrusion or attempt to intrude in the house. 8051 has been extensively used in past projects. However, this paper looks into incorporation of an ANDROID application which interprets the message a mobile device receives on possible intrusion and subsequently a reply (Short Message Service) SMS which triggers an alarm/buzzer in the remote house making others aware of the possible intrusion. Controlling home appliances remotely with mobile applications have started becoming quite popular due to the exponential rise in use of mobile devices. There have been so many applications that exploit the use of GSM/GPRS facility of the handset [4]. Mobile handsets today are essentially handheld computers with integrated mobile radio communication capabilities. With increasing usage of GSM, network services are expanded beyond speech communication to incorporate many other custom applications, machine automation and machine to machine communication. This paper discusses an approach where an authorized remote mobile 
user receives an SMS when a third party tries to enter his house in a remote location. The minimum requirement at the user end is that the mobile device should have an ANDROID OS. ANDROID is a java based operating system which runs on the Linux 2.6 kernel. It's lightweight and full featured. ANDROID applications are developed using Java and can be ported to new platform easily thereby fostering huge number of useful mobile applications [6]. A hardware circuit with a switch and a GSM modem embedded should be installed and connected to the door of the house. When the intruder tries to open the door, the switch triggers an interrupt and subsequently sends a signal into the microcontroller which subsequently triggers the GSM modem to transmit a warning SMS into already registered number in the modem. The SMS on the users' end is interpreted by the ANDROID Application and if it finds that the SMS is from the designated number; the application immediately informs the person with a frequent pop-up menu. If the user positive acknowledge the pop-up in 1 minute, an acknowledgement is send back to the remote GSM modem. The modem will output an interrupt to the microcontroller and the microcontroller will subsequently trigger an alarm. If the user fails to acknowledge in the defined time interval, an automatic positive acknowledgement will be sent by the application to the modem and the activities follow.[7]

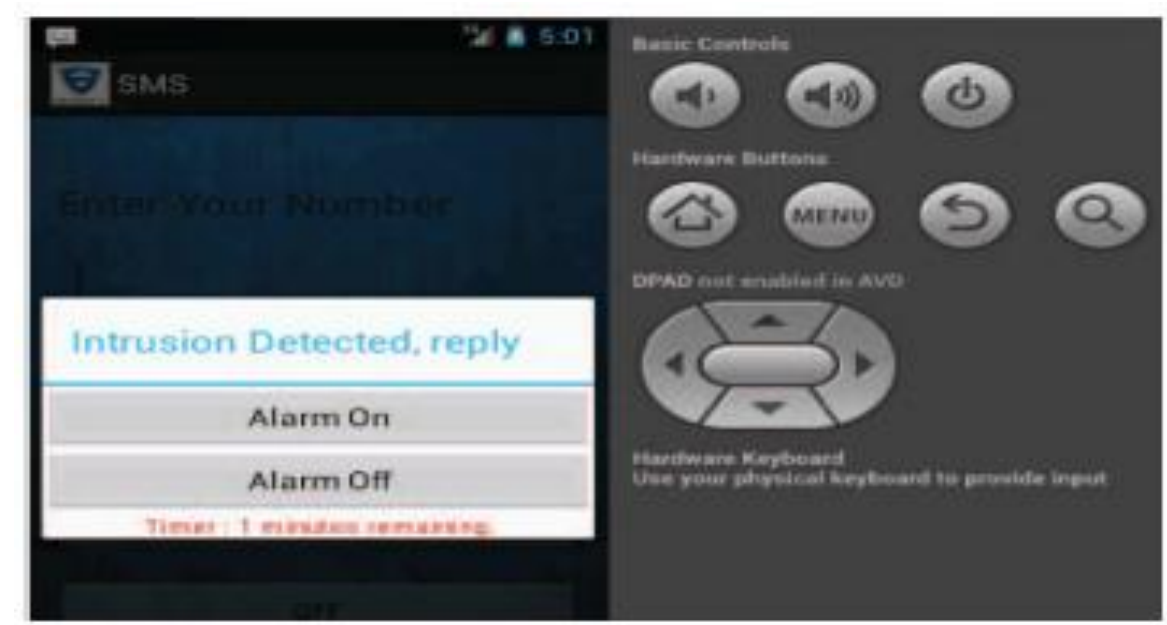

Figure 8 : Pop up on receiving an intrusion SMS from the saved number.

\section{Conclusion}

So, in this discussion we can conclude by saying that mobile phones can be launched with many features (with almost all the functions discussed). Furthers the concepts or the ideas that can be incorporated on the mobile phones can be extended and can be implemented keeping in consideration the view of economic and technical feasibility. These types of applications as stated earlier can be of immense help for all those people using these applications.
[1]. http://www.britishnutritions.in/activity-calculator.html
[2]. http://www.britishnutritions.in/bmi-calculator.html
[3]. A. Alheraish, Member, IEEE "Design and Implementation of Home Automation System", IEEE Transactions on Consumer Electronics, Vol. 50, No. 4, pp. 1087-1092, November 2004.
[4]. http://www.britishnutritions.in/body-fat-calculator.html
[5]. Dhruba Jyoti Gogoi, Rupam Kumar Sharma, "Android Based Emergency Alert Button", In International Journal of Innovative Technology and Exploring Engineering (IJITEE), ISSN: 2278-3075, Volume-2, Issue-4, pp. 26-27, March 2013.
[6]. http://www.britishnutritions.in/bmr-calculator.html
[7]. Rupam Kumar Sharma, Ayub Mohammad, Himanka Kalita, Dhiraj Kalita, “Android Interface based GSM Home Security System”, IEEEXplore.
[8]. https://play.google.com/store/apps/details?id=com.sharecare.sos\&hl= en
[9]. http://www.britishnutritions.in/calorie-calculator.html
[10]. Professional Android Application Development by Reto Meier.

\section{Authors Profile}

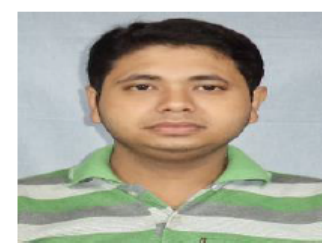

BHASKAR KAMAL BAISHYA received his B.Tech Degree in Computer Science and Engineering from, Assam Don Bosco University, Assam. Now he is an M.Tech final year student in the Department of Computer Science and Engineering, North Eastern Regional Institute of science and Technology, Nirjuli, Arunachal Pradesh. His research interest includes Embedded Systems and Cloud Computing. 